\title{
Hallazgos hemodinámicos en pacientes eclámpticas
}

\author{
Juan Carlos Sabogal*
}

\begin{abstract}
RESUMEN: La hipertensión inducida por el embarazo es la principal causa de muerte materna y fetal en nuestro hospital. La literatura describe diferentes patrones hemodinámicos que oscilan desde bajo gasto cardíaco y resistencia sistémica elevada; hasta más recientemente, alto gasto cardíaco y resistencia sistémica disminuida en un estadio subclínico. El objetivo de este estudio es identificar el patrón existente al momento del ingreso a la unidad de cuidados intensivos (UCI) para establecer la diferencia, si la hay, entre los patrones entre pacientes sobrevivientes y los que fallecieron y aclarar la correlación entre los parámetros de llenado ventricular. Se revisó la hemodinamia de 15 pacientes eclámpticas enviadas a la UCI para apoyo hemodinámico y/o ventilatorio. Se registraron el índice sistólico (IS), índice de trabajo ventricular (ITV), presión pulmonar media (PPM), índice de resistencia sistémica (IRS), índice de resistencia pulmonar (IRP), presión capilar pulmonar en cuña (PCP), y presión venosa central (PVC) al ingreso y durante su estancia en UCI. La primera medida reveló un promedio de IS=34.43 ml. ${ }^{2} . \mathrm{seg}, \mathrm{ITV}=3.09 \mathrm{gm} . \mathrm{m}^{2} . \mathrm{seg}, \mathrm{PPM}=31 \mathrm{mmHg}, \mathrm{IRS}=2594.93 \mathrm{din} . \mathrm{seg} / \mathrm{cm} . \mathrm{m}^{2}, \mathrm{IRP}=376.43 \mathrm{din} . \mathrm{seg} / \mathrm{cm} 5 . \mathrm{m}^{2}, \mathrm{PCP}=$ $16.60 \mathrm{mmHg}, \mathrm{PVC}=5.9 \mathrm{~cm} \mathrm{H2O}$. No hubo diferencia estadísticamente significativa entre las pacientes que sobrevivieron y las que fallecieron, sin embargo, las pacientes que sobrevivieron tuvieron tendencia a mantener mayores IS y menores PPM, IRS y PVC. Se encontró una fuerte correlación entre la PVC y la PCP. Se concluye que las pacientes eclámpticas en esta serie mostraron un patrón con función ventricular disminuida, presiones de llenado aumentadas y resistencias elevadas. No hubo diferencia entre los patrones hemodinámicos entre las pacientes que sobrevivieron y las que fallecieron y finalmente la fuerte correlación entre la PVC y la PCP sugieren la utilidad de la primera en la determinación de la precarga en estas pacientes.
\end{abstract}

PALABRAS CLAVES: Hemodinamia, eclampsia.

SUMMARY: Pregnancy induced hipertension is the main cause of both maternal and fetal death in our hospital. Literature describes different hemodynamic patterns ranging from low cardiac output and high peripheral resistance, to more recently, high cardiac output and low peripheral resistance in a subclinical stage. The objetive was to identify the pattern present at admission to the intensive care unit (I.C.U.), to establish a difference if any, between live and dead patients patterns and to clarify the correlation between ventricular filling pressure parameters. The hemodynamics of 15 eclamptic patients sent to the I.C.U. for hemodynamic and ventilatory support is revised. Sistolic index (IS), ventricular perfor mance index (VPI), mean pulmonar prressure (MPP), sistemic resistance index (SRI), pulmonary resistance index (PRI), pulmonary capilary wedge pressure (PCWP) and central venous pressure (CVP) were recorded at admission and during management AT I.C.U. The first measure revealed an average of $I S=34.43 \mathrm{ml} . \mathrm{m}^{2} . \mathrm{seg}, \mathrm{VPI}=3.09 \mathrm{gm} . \mathrm{m}^{2} . \mathrm{seg}, \mathrm{PP}=31 \mathrm{mmHg}, \mathrm{SRI}=$ $2594.93 \mathrm{din} . \mathrm{sec} / \mathrm{cm} 5 . \mathrm{m}^{2}, \mathrm{PRI}=376.43 \mathrm{din} . \mathrm{sec} / \mathrm{cm} 5 . \mathrm{m}^{2}, \mathrm{PCWP}=16.60 \mathrm{mmHg}, \mathrm{CVP}:=5.9 \mathrm{~cm} \mathrm{H} 2 \mathrm{O}$. No significant difference was found bet ween hemodynamic patterns of patients who lived and those who died. However, survival patients tended to have a higher si and lower MPP, SRI and CVP when compared with dead patients. A strong correlation was found between CVP and PCWP. It is concluded that eclamptic patients in this series showed a pattern of dismished ventricular function, elevated ventricular filling pressures and elevated both pulmonary and sistemic resistances. There was no difference between patterns of live and dead patients and finally, the strong correlation between $\mathrm{CVP}$ and PCWP suggests CVP may be useful assessing cardiac preload.

KEY WORDS: Hemodynamics, eclampsia.

La toxemia se constituye en nuestro medio en la mayor causa de muerte materna desde 1990 (1). Alrededor del mundo su incidencia se ha calculado desde un 0.51 hasta en un $38.4 \%$ (2), dependiendo de los criterios empleados. La enfermedad se caracteriza por su pleomorfismo clínico, curso inesperado y la ausencia de un marcador biológico (3). El diagnóstico se considera en pacientes con más de 20 semanas de embarazo, que presenten edema, proteinuira e hipertensión (4-6). Hemodinámicamente, las pacientes con hipertensión inducida por el embarazo (HIE), estudiadas por Wallemburg et al, mostraron un patrón de alta resistencia periférica y bajo gasto cardíaco (7). Hankins et al, en pacientes eclámpticas realizó restricción hídrica encontrando disminución de la presión capilar pulmonar (8). Pridjian et al, en pacientes con HIE, encontraron disminución del gasto cardíaco y resistencia vascular aumentada con respec-

Instructor asociado, Universidad Nacional de Colombia, Instituto Materno Infantil, Departamento de Ginecobstetricia. Santafé de Bogotá. to a embarazadas normales (9). La alta resistencia periférica se confirmó con estudios bioquímicos que mostraron disminución de la excreción de metabolitos estables de prostaciclina (10). Gant et al, demostraron mayor depuración en el metabolismo de dihidroisoandrosterona, función placentaria, en pacientes con HIE con respecto a embarazadas normales. Otros investigadores han encontrado aumento del gasto cardíaco en pacientes con HIE (12). Estudios longitudinales, mostraron un patrón de alto gasto cardíaco y resistencias periféricas menores en pacientes con HIE comparadas con embarazadas normales (13). Phelan et al, propusieron como modelo un "continum" fisiopatológico desde un estado subclínico de alto gasto y baja resistencia, hasta un estado clínico de bajo gasto y alta resistencia (14). Quizás esto explique los resultados favorables al tratar estas pacientes con Betabloqueadores (15). Así mismo, la compresión de los fenómenos hemodinámicos, acaso disminuya la frecuencia de complicaciones en estas pacientes, entre ellas, el edema pulmonar (16), frecuente en nuestro medio. El presente estudio pretende identificar la tendencia 
hemodinámica de nuestras pacientes eclámpticas, establecer las diferencias entre aquellas que sobrevivieron y las que fallecieron y finalmente, dentro del esquema encontrado, correlacionar la presión venosa central (PVC) y la presión capilar pulmonar en cuña (PCP), para definir la utilidad de la primera en el manejo de estas pacientes.

\section{Material y métodos}

Se revisaron los registros de ingreso a la Unidad de Cuidado Intensivo (UCI) del Hospital San Juan de Dios, de los últimos 10 años y se seleccionaron las pacientes con diagnóstico de ingreso de toxemia. Los criterios de inclusión fueron la presencia de proteinuria de más de $100 \mathrm{mg} /$ $\mathrm{dl}$ en muestra al azar o de $300 \mathrm{mg} / \mathrm{dl}$ en orina de 24 horas, Tensión Arterial mayor o igual a $140-90$ y la presencia de edema pretibial, que cursaron con eclampsia y que fueron remitidas desde el Instituto Materno Infantil a la UCI para soporte ventilatorio y/o hemodinámico. Los criterios de exclusión del estudio fueron la presencia de enfermedad vascular crónica, epilepsia, enfermedad renal, de la coagulación, hepática o del colágeno previas a la gestación y las pacientes que en algún momento de su evolución presentaron compromiso séptico y aquellas que no reunían los criterios de inclusión (4). Como datos de interés, se registró la edad, estancia en UCI, complicaciones y estado al egreso. Los parámetros hemodinámicos considerados fueron: Indice Sistólico (IS), Presión Pulmonar Media (PPM), Indice de Resistencia Arterial Pulmonar (IRAP), Indice de Resistencia Arterial Sistémica (IRAS), Indice de Performance Ventricular (IPV), Presión Venosa Central (PVC) y Presión Capilar Pulmonar en Cuña (PCP), tal y como se definen en la Tabla 1, medidos a intervalos de 8 horas, con instalación al ingreso a UCI, de un catéter de Swan-Ganz (Arrow Corp.) (17), vía vena subclavia, y con un monitor Horizon-4000, que utiliza el método de termodilución para cálculo del gasto cardíaco. Se registró y promedió cada uno de los parámetros al ingreso, las pacientes que fallecieron fueron separadas de las que sobrevivieron y se obtuvieron gráficas de tendencia de cada parámetro en el tiempo, comparando estas dos poblaciones. Finalmente, se realizó una gráfica comparativa de la PVC y la PCP en el tiempo. El análisis estadístico se centró en el análisis de varianza usual (anova), apoyado en métodos no paramétricos auxiliares de KruskalWallis test (Wilcoxon), Van der Waerden test y Savage test, con el objeto de comparar los parámetros hemodinámicos entre las pacientes vivas y las que fallecieron en cada

\section{Tabla 1}

medición. Para evaluar la asociación entre PVC y PCP, se realizó un análisis de correlación utilizando el coeficiente r (rho) de Sperman en cada medición. Para aplicar los métodos estadísticos enunciados, se usó el paquete SAS (Statistical Analysis System).

\section{Resultados}

15 pacientes cumplieron los criterios de inclusión. El promedio de edad fue de 25 años. La estancia promedio fue de 7.4 días. La mortalidad fue del $43.8 \%(n=7)$. Las complicaciones más comunes fueron la insuficiencia renal aguda en el $76 \%(n=13)$, edema pulmonar en el $35 \%(n=6)$, coagulación intravascular diseminada, síndrome de dificultad respiratoria del adulto y síndrome HELLP, en el $23.5 \%$ (n=4) cada uno.

Las variables hemodinámicas de la primera medición al ingreso a UCI, se encuentran resumidas en la Tabla 2. Las gráficas estratificadas (vivas/muertas) de cada variable en función del tiempo, se muestran en la gráfica 1.

$\Gamma$ ningún caso, la diferencia entre los niveles medios de cad variable, entre las pacientes vivas y las que sucumbieron le estadísticamente significativa para la estratificación rea] ada (vivas/muertas) a pesar de lo cual es factible un aná is de los datos obtenidos (ver gráficas 1 y 2): el IS tuvo en ? neral, un valor mayor en las pacientes que sobrevivieron a PPM, fue mayor en las pacientes que murieron. El IRF endió a ser mayor en las pacientes que fallecieron. El IRS ie comportó de modo muy similar entre los dos grupos. El I $\checkmark$, no mostró diferencia entre las pacientes estudiadas. La ] C, se mostró elevada en las pacientes que sucumbieron compararlas con las pacientes que sobrevivieron. $\mathrm{La}$ PCI se comportó de un modo muy similar en los dos gru s. Finalmente, la gráfica de coeficientes de correlaciól sara cada medición, entre la PVC y la PCP, muestra vals ss altos que son estadísticamente significativos. Los valc is de P fueron en general menores a 0.005. (Ver gráfica $3)$, : el coeficiente de determinación fue de 0.64.

Tabla 2

\begin{tabular}{|c|c|c|c|c|c|c|c|}
\hline \multirow[b]{2}{*}{ Vari } & \multirow[b]{2}{*}{ e } & \multirow[b]{2}{*}{ Mínimo } & \multirow[b]{2}{*}{ Máximo } & \multirow[b]{2}{*}{ Promedio } & \multirow[b]{2}{*}{ SD } & \multicolumn{2}{|c|}{$\begin{array}{l}\text { Valores } \\
\text { Normales }\end{array}$} \\
\hline & & & & & & Mínimo & Máximo \\
\hline IS1 & 14 & 25 & 49 & 34.43 & 6.69 & 35 & 50 \\
\hline PPN & 15 & 11 & 49 & 31.00 & 9.71 & 12 & 20 \\
\hline RP & 14 & 141 & 843 & 376.43 & 204.93 & 250 & 250 \\
\hline RSI & 14 & 1666 & 5184 & 2594.93 & 924.57 & 1800 & 2200 \\
\hline PV1 & 14 & 12 & 56 & 30.93 & 12.60 & 4 & 8 \\
\hline PVC & 15 & 1 & 25 & 14.27 & 5.90 & 6 & 12 \\
\hline PCP & 15 & 3 & 36 & 16.60 & 7.92 & 6 & 12 \\
\hline
\end{tabular}

\section{Disc sión}

I edad promedio fue de 25 años, lo que hace improbable 1 resencia de un componente hipertensivo crónico. La estal ia promedio (7 días), está acorde con la habitual histc a natural de la enfermedad evolucionando rápidamen hacia la mejoría o hacia la muerte. La mortalidad de la pc lación estudiada (43.8\%), no es comparable dado que no e. iten datos en poblaciones similares. Acaso, el porcen- 
Grafica 1.

PERFORMANCE VENTRICULAR

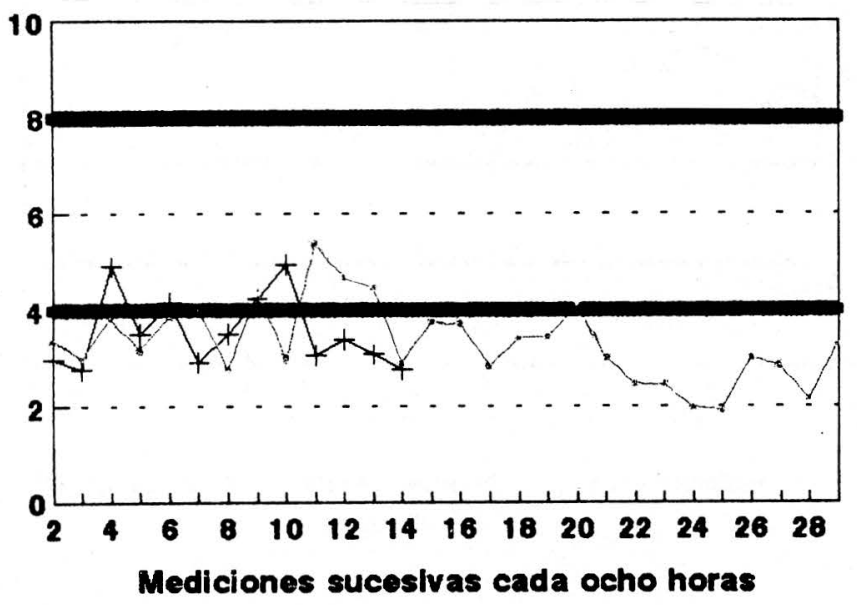

- Pacientes vivas + Pacientes muertas - Rango Normal
PRESION CAPILAR PULMONAR

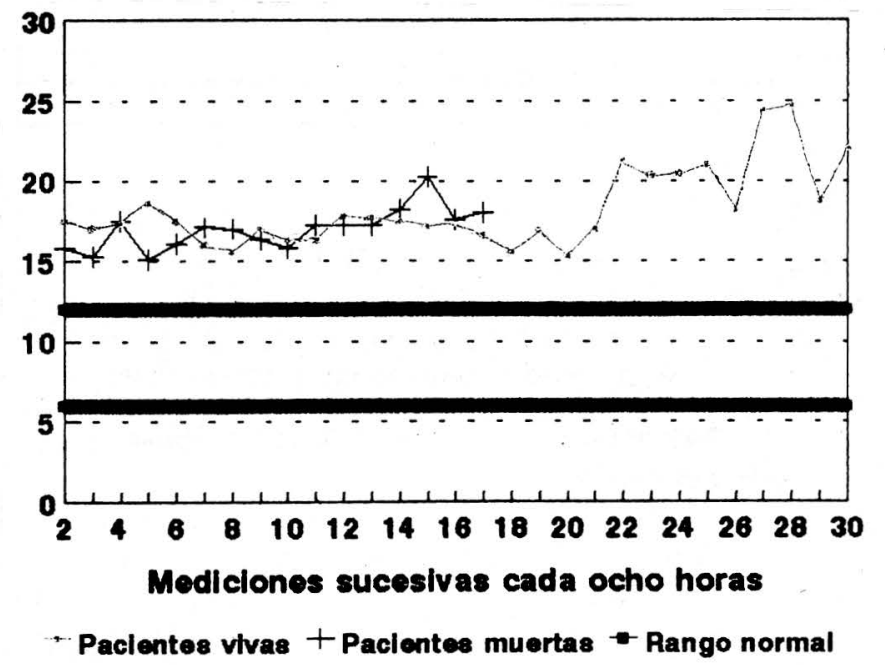

INDICE SISTOLICO

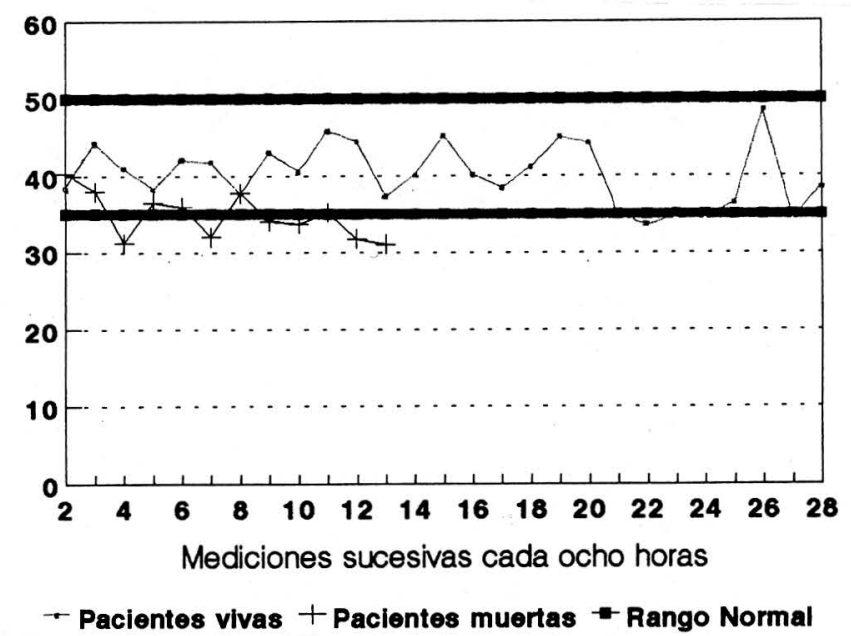

Grafica 2.

PRESION PULMONAR MEDIA

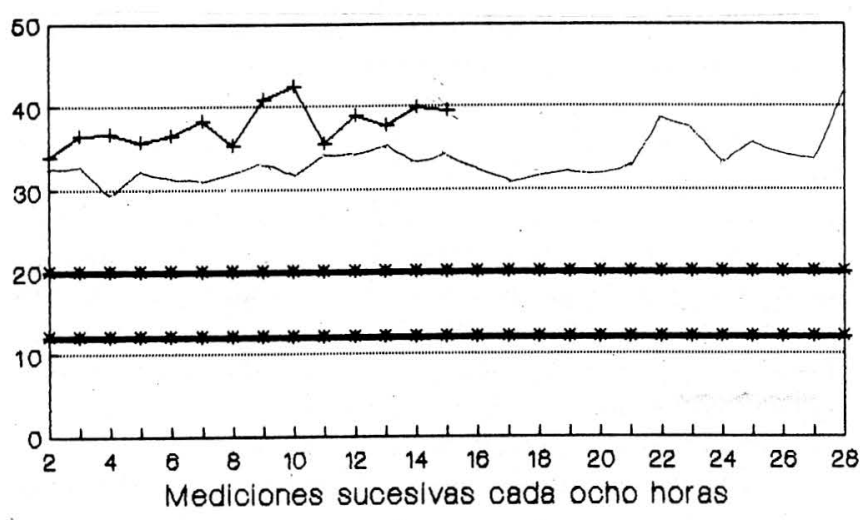

- Pacientes vivas + Pacientes muertas - Pango normal
INDICE DE RESISTENCIA PULMONAR

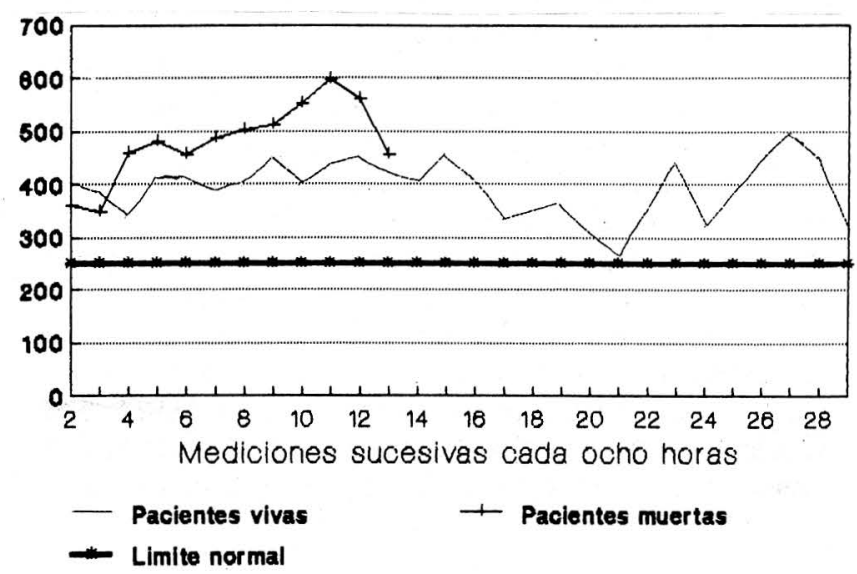


INDICE DE RESISTENCIA SISTEMICA

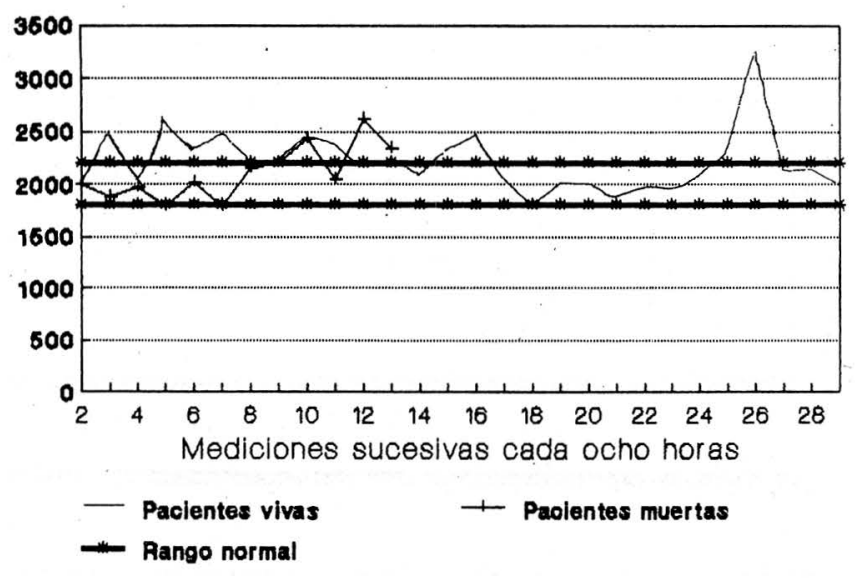

PRESION VENOSA CENTRAL

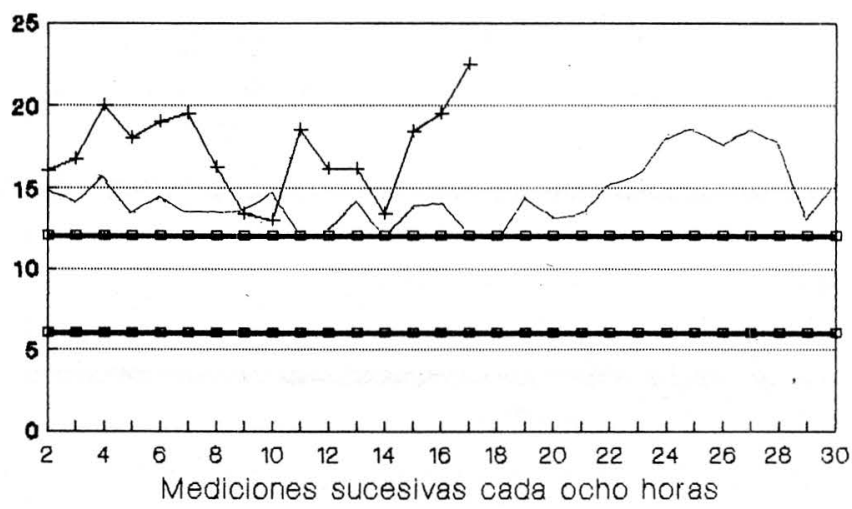

- Pacientes vivas + Pacientes muertas - Rango normal

Grafica 3 .

CORRELACION PVC-PCP

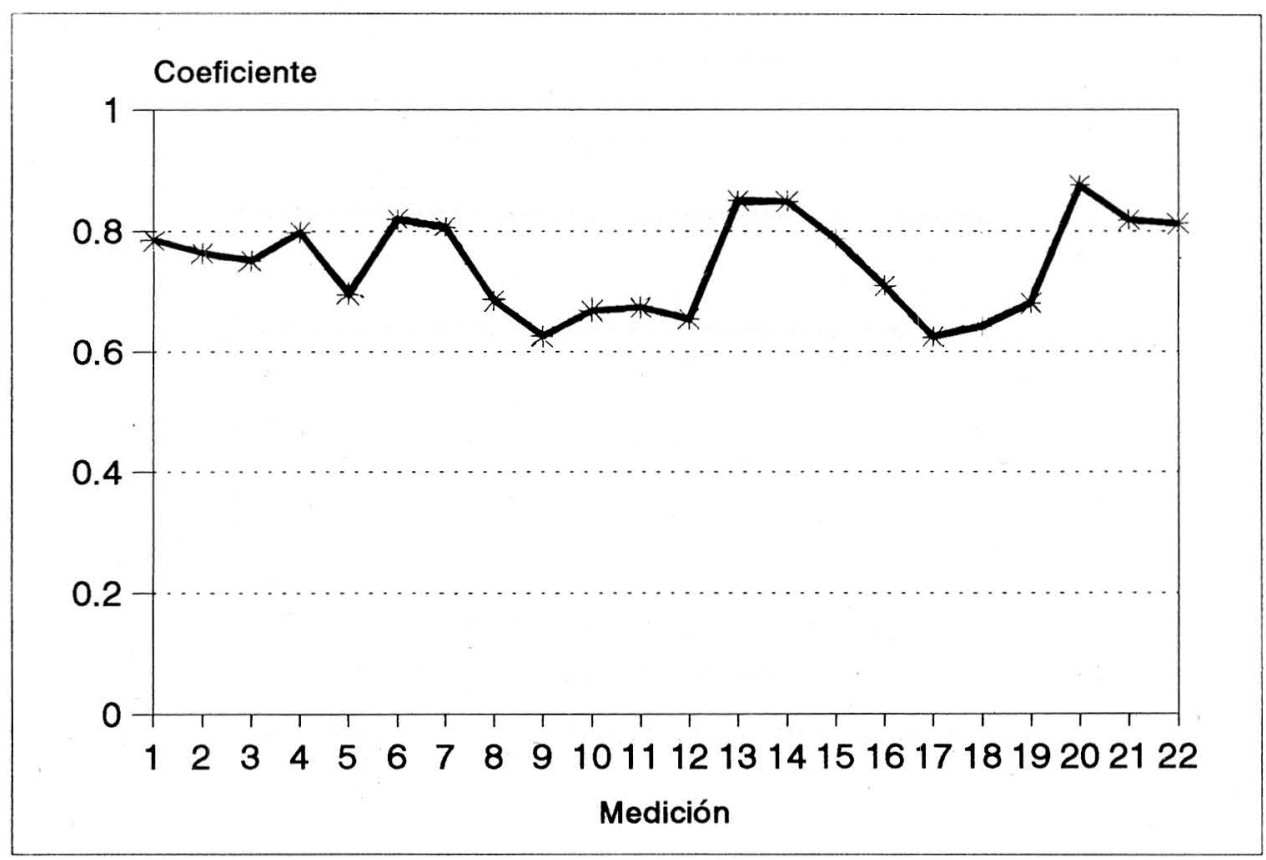

taje disminuyera si el traslado a UCI fuera más temprano en el curso de la enfermedad. Las complicaciones encontradas, son las mismas que han sido reportadas en otras series (3-5, 18-19), lo cual podría relacionarse no sólo a la naturaleza de la enfermedad per sé, sino al deficiente control de proceso patológico. Tales complicaciones, probablemente expliquen la mortalidad de esta muestra.

Los datos de la primera lectura, muestran un patrón hemodinámico caracterizado por unos parámetros de función cardíaca (IS, IPV) ubicados en el extremo inferior de los valores asumidos como normales para el adulto sano, no grávido, y son comparables a lo previamente encontrado por Mabie et al (20) y Cotton et al (21), en los cuales el IS obtenido fue de 48 y $44 \mathrm{ml} . \mathrm{m}^{2}$.seg., respectivamente. La diferencia probablemente estribe en que las pacientes de esta serie presentaron serias complicaciones médicas que no se encontraban en los dos estudios citados. El IS hallado es necesariamente bajo si se considera que en la paciente embarazada normal, el gasto cardíaco aumenta hasta en un $40 \%$ con respecto al adulto no embarazado (22). Los parámetros pulmonares (PPM, IRP), se mostraron elevados con respecto a las cifras normales y aún en la PPM hallada por Marbie et al y Cotton et al, de 15 y $17 \mathrm{mmHg}$. El IRP del presente estudio fue encontrado al ingreso a UCI en un promedio de 376.43 din.seg./ $\mathrm{cm} 5 . \mathrm{m}^{2}$, comparándose con el hallado por Mabie et al, de 121 y Cotton et al de 127 din.seg./ $\mathrm{cm} 5 . \mathrm{m}^{2}$, hallazgo en directa relación a la PPM, y a la frecuencia del edema pulmonar en esta serie. El IRS, fue 
encontrado en un promedio de 2594.93 din.seg./cm5. $\mathrm{m}^{2}$, cifra muy elevada con respecto a lo hallado en embarazadas normales de 800 a 1200 din.seg./cm5.m². Este hallazgo evidencia no sólo la severidad de la enfermedad, sino el mal control hemodinámico de este parámetro que seguramente perpetua el daño vascular ensombreciendo más el pronóstico. La PVC se halló en $14.27 \mathrm{cmH} 2 \mathrm{O}$, más elevada que la hallada por Mabie et al y Cotton et al de 4.8 y $4.0 \mathrm{cmH} 2 \mathrm{O}$. La PCP se encontró en $16.60 \mathrm{mmHg}$, que luce elevada al compararse con la previamente encontrada (20-22). Estas cifras seguramente derivan de una función ventricular disminuida ya evidenciada en el análisis del IS y el IPV். En general, el perfil hemodinámico de las pacientes eclámpticas en la presente serie estuvo caracterizado al ingreso a UCI, por un patrón de altas resistencias pulmonares y periféricas asociadas a presiones de llenado aumentadas y función ventricular disminuida. Dicha situación se reconoce al final del espectro de lo que Phelan et al dio en llamar un "continuum" fisiopatológico a partir de un estado subclínico de HIE, caracterizado por alto gasto y bajas resistencias (14). El manejo farmacológico previo de las pacientes antes del arribo a UCI, puede influenciar este perfil, pero dicha intervención es inevitable.

Las gráficas de cada parámetro estratificadas en una población de sobrevivientes comparada con una población de fallecidas no mostraron diferencias estadísticamente significativas entre los dos grupos, a pesar de lo cual puede teorizarse que aquellas pacientes que sobrevivieron presentaron un IS más alto, asociado a una PPM, IRP y PVC más bajos al compararlas con las pacientes que fallecieron. En el futuro, quizás sea posible establecer la presencia de parámetros hemodinámicos prioritariamente controlables y de valor pronóstico en el manejo de estas pacientes.

Finalmente, llama la atención la fuerte correlación entre la PVC y la PCP, encontrada en esta serie, lo cual se contrapone a lo previamente hallado por Cotton et al (23). Este hallazgo hipotéticamente podría sugerir que la PVC, sería un parámetro aproximado en la determinación de la PCP, mucho más dispendiosa desde el punto de vista clínico. En el futuro, los estudios longitudinales iniciados en épocas tempranas del embarazo así como la aplicación de técnicas no invasivas al estudio hemodinámico de las pacientes toxémicas, lograrán dilucidar muchos de los interrogantes que aún quedan por aclarar a este respecto.

\section{Agradecimientos}

A la asesoría prestada por los Drs: Octavio Martínez de la Unidad de Cuidados Intensivos del Hospital San Juan de Dios, Santafé de Bogotá, profesor Humberto Mayorga del departamento de Estadística de la Universidad Nacional de Colombia y Hernando Gaitán Profesor Asociado. Departamento de Ginecobstetricia, Universidad Nacional de Colombia.

\section{BIBLIOGRAFIA}

1. Boletín informativo de Comité de Mortalidad del Instituto Materno Infantil de Santafé de Bogotá, marzo de 1993.

2. Plouin $\mathrm{PF}$ et al. Fetal grouth retardation in gestational hypertension: relations with blood pressure levels and the time of onset of hypertension. Eur J. Obstet. Gynecol. Reprod. Biol. 1983; 16(4): 253-262.

3. Arias F. Practical guide to high-risk pregnancy and delivery. Second edition, Mosby Year Book, 1993.

4. Grupo de estudio de la O.M.S. sobre transtornos hipertensivos del embarazo. Informe de un grupo de estudio de la O.M.S., serie de informes técnicos. Ginebra, septiembre de 1985.

5. Creasy RK., Resnik R. Maternal-fetal medicine:principles and practice. Second edition. W.B. Saunders Company 1989.

6. Sibai BM. Pitfalls in the diagnosis and management of Preeclampsia. Am. J. Obstet. Gynecol. 1988; 159: 1-5.

7. Groenendijk R., Trimbos JB., Wallemburg HC. Hemodynamic measurement in preeclampsia: Preliminary observations. Am. J. Obstet. Gynecol. 1984; 150: 232-236.

8. Hankins GD., Wendel GD., Cunningham FG., Leveno KJ. Longitudinal evaluation of hemodynamic changes in eclampsia. Am. J. Obstet. Gynecol. 1984; 150: 508-512.

9. Pridjian G., Lang R., Feldman T., Borow K., Lindheimer MD. Cardiac output (CO) and load independient left ventricular (LV) contractility (CTR) in preeclampsia (PIH) assessed noninvasely. En: Mashall D., Lindheimer MD, Katz AI. Preeclampsia: Pathophysiology, diagnosis, and management. Am. Rev. Med. 1989; 40: 233-250.

10. Ylikokala O., Pekonen F., Viinikka L. Renal prostacyclin and thromboxane in normotensive and preeclamptic pregnant women and their infants. J Clin Endocrinol Metab 1986; 63: 1307.

11. Gant NF., Madden JD., Siiteri PK., MacDonald PC. Fetal-Maternal interrelationships: a secuential study of the metabolism of dehidroisoandrosterone sulfate in primigravid pregnancy. In: Proccedings of the fourth international congress of endocrinology. Amsterdam: International congress series No. 273. Excerpta Medica $1972 ; 1026$.
12. Hilary et al. Citado en: Easterling TR., Benedetti TJ. Preeclampsia: a hyperdinamic disease model. Am. J. Obstet. Gynecol. 1989; 160: 1447-1453.

13. Easterling TR., Benedetti TJ., Schmucker BC., Millard SP. Maternal hemodynamics in normal and preeclamptic pregnancies: A longitudinal study. Obstet. Gynecol. 1990; 76: 1061.

14. Phelan JP., Yurth PA. Severe preeclampsia. I. Peripartum hemodynamic observations. Am. J. Obstet. Gynecol. 1982; 144: 17.

15. Eliahou HE et al. Propanolol for the treatment of hypertension in pregnancy. British J. Obstet. Gynecol. 1878; 86: 431-436.

16. Sibai BM., Mabie BC., Harvey CJ., González AR. Pulmonary edema in severe preeclampsia-eclampsia analysis of thirty-seven consecutive cases. Am. J. Obstet. Gynecol. 1987; 156: 1174-1179.

17. Swan HJC., Ganz W., Forrester J et al. Catheterization of the heart in man with use of a flow directed ballon-tiped catheter. New. Engl. J. Med. 1970; 283: 447.

18. Strauss RG., Keefer JR., Burke T., Civetta JM. Hemodynamic monitoring of cardiogenic pulmonary edema complicating toxemia of pregnancy. Obstet. Gynecol. 55; 2: 170-174.

19. Benedetti TJ., Kates R., Williams V. Hemodynamic observations in severe preeclampsia complicated by pulmonary edema. Am. J. Obstet. Gynecol. 1985; 152: 330-334.

20. Mabie WC., Ratts TE., Sibai BM. The central hemodynamics of severe preeclampsia. Am. J. Obstet. Gynecol. 1989; 161: 1443-1448.

21. Cotton DB., Gonik B., Dorman KF. Cardiovascular alterations in pregnancy-induced hipertension: acute effects of magnesium sulfate. Am. J. Obstet. Gynecol. 1984; 148: 162-165.

22. Bader RA., Bader ME., Rose DJ., Braunwald E. Hemodynamics at rest and during exercise in normal pregnancy as studied by cardiac catheterization. J. Clin. Invest. 1955; 34: 1524-1536.

23. Cotton DB., Gonik B., Dorman K., Harrist R. Cardiovascular alterations in severe pregnancy-induced hypertension: Relationship of central venous pressure to pulmonary capillary wedge pressure. Am. J. Obstet. Gynecol. 1985; 151: 762-764. 